\title{
Diet-induced Atherosclerosis in Mice Heterozygous and Homozygous for Apolipoprotein E Gene Disruption
}

Sunny H. Zhang, Robert L. Reddick, Bryan Burkey, ${ }^{\star}$ and Nobuyo Maeda

Department of Pathology, School of Medicine, University of North Carolina at Chapel Hill, North Carolina 27599-7525; and * Sandoz

Research Institute, East Hanover, New Jersey 07936

\begin{abstract}
With the aim of establishing whether a genetically reduced capability of producing apolipoprotein $\mathbf{E}$ (apo E) can affect atherogenesis, we have compared the consequences of dietary stress on normal mice and on mice heterozygous or homozygous for a disrupted apo $E$ gene. A dramatically accelerated development of lesions occurred in the vasculature of the homozygous mutants as a result of feeding an atherogenic diet for $12 \mathrm{wk}$, and extensive deposition of lipidfilled macrophages was found outside the cardiovascular system. In nine heterozygotes fed the atherogenic diet for $12 \mathrm{wk}$, the amount of apo $E$ in their total plasma lipoproteins increased to a level comparable to normal, but all nine developed much larger foam cell lesions in their proximal aorta than those found in 3 of 9 normal mice fed the same diet. The other six normals had no lesions. Our study demonstrates that heterozygous mice with only one functional apo $\mathbf{E}$ gene are more susceptible to diet-induced atherosclerosis than are normal, two-copy mice. Genetically determined quantitative limitations of apo $E$ could, therefore, have similar effects in humans when they are stressed by an atherogenic diet. (J. Clin. Invest. 1994. 94:937-945.) Key words: high-fat, high-cholesterol diet • gene targeting • macrophage - hypercholesterolemia • apo E-deficiency
\end{abstract}

\section{Introduction}

Atherosclerosis is a common and insidious disease which accounts for most of the morbidity and mortality seen in humans with coronary heart disease and stroke. There are numerous risk factors for developing atherosclerosis (1) and the importance of the combined effects of genetic and environmental factors has been suggested by numerous studies (2). Dissection of the relative contribution of the various genetic and environmental factors is however needed for a more complete understanding of the disease and for developing more effective therapies. Due to the limitations and difficulties of conducting such studies in humans, a variety of animal models have been used, such as rabbits, pigs, dogs, pigeons, and primates. Mice have been the most used for genetic studies because of the availability of numerous well characterized inbred strains and the relative ease

Address correspondence to Dr. Nobuyo Maeda, Department of Pathology, CB\#7525, Brinkhous-Bullitt, University of North Carolina at Chapel Hill, Chapel Hill, NC 27599-7525.

Received for publication 19 January 1994 and in revised form 11 May 1994.

J. Clin. Invest.

(C) The American Society for Clinical Investigation, Inc.

0021-9738/94/09/0937/09 \$2.00

Volume 94, September 1994, 937-945 of mapping relevant genes in mice. Their value has been further increased by advances in molecular biological technologies which now allow the mouse germline to be manipulated. Mice either overexpressing or carrying genetically inactivated genes have been produced in the past few years (for reviews see references 3-7). Such mice should be important for elucidating the interaction between discrete genetic components and controlled environmental factors in the process of atherogenesis.

Mice are naturally resistant to atherosclerosis, and diets containing high fat and high cholesterol have been required to produce atherosclerotic lesions in normal inbred strains of mice. We and others have found, however, that apolipoprotein (apo) E-deficient mice generated by gene targeting $(8,9)$ have spontaneously elevated plasma cholesterol levels, even when fed regular chow and develop severe atherosclerotic lesions very similar to those present in humans. In contrast, mice heterozygous for the mutant gene did not show abnormalities in lipid metabolism when fed regular chow, and no arterial lesions were detected in them (10)

It has been well established, in human (11) and animal (12-16) studies, that diets high in cholesterol and saturated fatty acids can significantly elevate plasma cholesterol levels and increase the risk of atherosclerosis. The hypercholesterolemia and changes in lipoproteins induced by diet are associated with the high incidence of coronary artery disease seen in the U.S. population. apo $\mathrm{E}$ is one of the protein components of all lipoproteins except low density lipoprotein (LDL) in humans. It serves as a ligand for the clearance of lipids from the plasma via receptor-mediated pathways in the liver. The exact role of apo $\mathrm{E}$ in diet-induced susceptibility to atherosclerosis is, however, still debatable. In some studies, different apo $\mathrm{E}$ genotypes have been associated with differences of plasma lipid levels in response to diet $(17-20)$, although in other studies no significant variation of plasma cholesterol levels in response to diet has been detected among individuals with different apo $\mathrm{E}$ alleles (21-22). To further understand the importance of apo $E$ in diet-induced atherosclerosis, we have here evaluated the responses of normal mice and of mice either homozygous or heterozygous for the genetically inactivated apo E gene to a diet high in cholesterol and saturated fat. Our study demonstrates that the null mutation of the apo $E$ gene, even in the presence of a normal allele, renders mice fed the atherogenic diet more susceptible to the development of atherosclerotic lesions than normal mice.

\section{Methods}

Animals and experimental design. Normal, heterozygous, and homozygous apo E mutant mice (8) were produced in house and used for the high-fat diet feeding studies. These mice have a mixed genetic background derived from the two inbred strains: C57BL/6 and 129. All the mice were maintained in a room illuminated from 7 AM to 7 PM. Two groups of studies were performed. The first experimental group contained mice 16-20 wk old: 7 normal mice ( 6 males, 1 female), 6 
heterozygotes ( 5 males, 1 female), and 10 mutant homozygotes (6 males, 4 females). In the second experimental group, mice 8 wk old were chosen: 4 normal mice ( 3 males, 1 female), 10 heterozygotes ( 7 males, 3 females), and 9 homozygotes ( 6 males, 3 females). Regular mouse chow (\#5012), which is low in fat and contains $4.5 \%$ (wt/wt) fat, $0.022 \%$ (wt/wt) cholesterol, was purchased from Ralston Purina (St. Louis, MO); in the following we refer to this chow as "regular chow." A mouse high-fat, high-cholesterol diet (TD 88051) containing $15.8 \%(w t / w t)$ fat, $1.25 \%(w t / w t)$ cholesterol, $0.5 \%(w t / w t)$ sodium cholate, was obtained from Teklad Premier (Madison, WI); in the following we refer to this diet "atherogenic diet." Food and water were provided with free access. Weight gain and plasma lipid levels were monitored monthly. Five homozygotes from the first experiment and three homozygotes from the second experiment were sacrificed for morphologic studies after $6 \mathrm{wk}$ of feeding the atherogenic diet; the remaining mice were killed after $12 \mathrm{wk}$ of feeding. An equal number of mice were fed regular chow for use as controls.

Collection of blood and plasma lipid assays. Blood from mice fasted for $16 \mathrm{~h}$ was collected by retroorbital bleeding into a tube containing EDTA, aprotinin and gentamicin as previously described (8). Plasma was harvested by centrifugation of the whole blood for $10 \mathrm{~min}$ at 12,000 $g$ at $4^{\circ} \mathrm{C}$.

Plasma lipid levels were assayed within $2 \mathrm{~d}$ of blood collection. Total plasma cholesterol and triglyceride for each individual sample were measured using a colorimetric method with commercially available kits (Sigma Chemical Co., St. Louis, MO). For measuring cholesterol levels of homozygotes, plasma samples were diluted 10 times in order to fit into the linear range of the test reading. High density lipoprotein (HDL) cholesterol was determined after precipitating the apolipoprotein B (apo B)-containing particles with magnesium/dextran sulfate (23).

Agarose gel electrophoresis. $1 \mu \mathrm{l}$ of pooled plasma from each group of mice was loaded with a Hamilton syringe onto a pre-made agarose gel film (Ciba Corning Diagnostics Corp., Palo Alto, CA). The gel was run at $9 \mathrm{~V} / \mathrm{cm}$, in Universal buffer (Ciba Corning Diagnostic Corp.) for $40 \mathrm{~min}$, then dried at $55^{\circ} \mathrm{C}$ for $15 \mathrm{~min}$, and stained with Oil Fat 7B (Ciba Corning Diagnostic Corp.).

Fast performance liquid chromatography. Fractionation of serum lipoproteins by superose- 6 gel permeation chromatography was performed with a robotics FPLC system as previously described (24). Briefly, the column matrix was equilibrated at $0.5 \mathrm{ml} / \mathrm{min}$ with running buffer ( $50 \mathrm{mM}$ Tris, $\mathrm{pH} 7.4,0.15 \mathrm{M} \mathrm{NaCl}$, and $0.01 \%$ sodium azide). $60 \mu \mathrm{l}$ of mouse serum was diluted with $160 \mu \mathrm{l}$ FPLC running buffer, then $200 \mu \mathrm{l}$ was injected followed by fractionation into forty $0.5 \mathrm{ml}$ fractions. $80 \mu \mathrm{l}$ of each fraction was assayed for cholesterol content using a colorimetric enzyme assay (Fast Cholesterol; Sclavo, Wayne, NJ).

Plasma apolipoprotein analysis. Total plasma lipoproteins were purified from pooled plasma by density $(d<1.21 \mathrm{~g} / \mathrm{ml})$ ultracentrifugation (25). After desalting and concentrating with Centricon 10 (Amicon Inc., Beverly, MA), protein concentrations of each sample were determined by modified Lowry assays (Bio-Rad Laboratories, Richmond, CA). A volume of each sample equivalent to $40 \mu$ l plasma was mixed with one-fifth volume of sample buffer $(312 \mathrm{mM}$ Tris- $\mathrm{HCl}, \mathrm{pH} 6.8$, $10 \%$ SDS, $25 \%$ glycerol, $2.5 \%$ beta-mercaptoethanol, and $0.005 \%$ bromphenol blue) and boiled for $5 \mathrm{~min}$ before loading onto a $16 \times 12 \times 0.15$ $\mathrm{cm}, 3-20 \%$ SDS-polyacrylamide gel (SDS-PAGE). After pre-running at $25 \mathrm{~mA}$ for $30 \mathrm{~min}$, protein bands were separated at $35 \mathrm{~mA}$ for $6 \mathrm{~h}$ and stained with Coomassie brilliant blue R-250.

Evaluation of atherosclerotic lesions. Mice fasted for $16 \mathrm{~h}$ were sacrificed with an overdose of Avertin (8). The abdominal and thoracic cavities were opened and the heart and vascular tree were perfused with phosphate buffered-paraformaldehyde $(4 \%, \mathrm{pH} 7.4)$ under physiological pressure. Segments of the proximal aorta and the portion of the heart containing the aortic sinus were embedded, sectioned and stained as previously described (10). Other tissue samples for light and electron microscopic studies were also prepared as previously described (10).

Four sections were used for morphometric evaluations. The first section measured was that in which the most proximal part of the ascending aorta had a round cross section. The second section was chosen

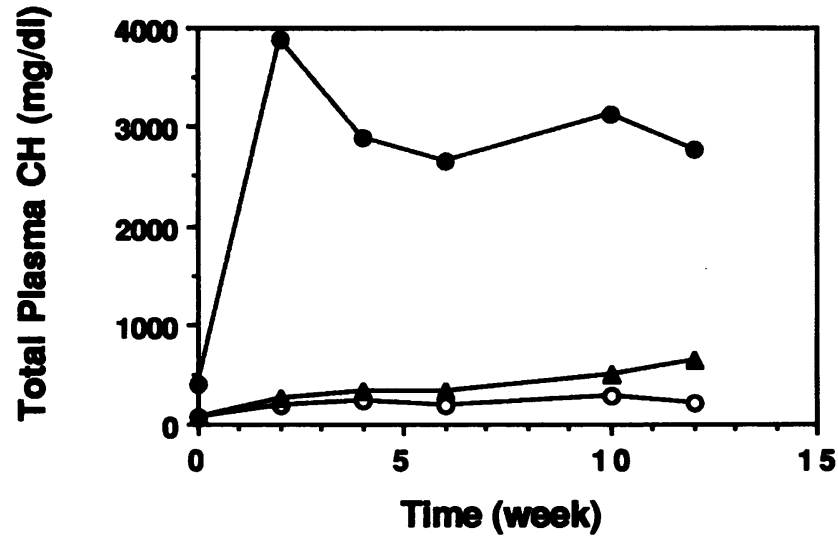

Figure 1. Changes of plasma cholesterol levels in normal $(0)$, heterozygous $(\Delta)$ and homozygous $(\bullet)$ mice during the course of the atherogenic diet feeding. Each point represents the mean plasma total cholesterol levels of 11 to 12 mice.

to include the valve attachment sites and the coronary ostia, but did not contain valve leaflets. The third section was characterized by the appearance of the valve leaflets as small nodules in the valve attachment sites. The fourth section was from a region where the valves appeared complete and were joined to their attachment sites. The average of the sizes of lesions in the above four sections was used to represent the lesion size of each animal. Morphometric evaluation of the lesion size was conducted using Image measure/IP IM 2500 Morphometry system (Phoenix Technology, Federal Way, MA).

Statistical analysis. Paired Student $t$ tests were performed for the plasma lipid levels before and $12 \mathrm{wk}$ after diet feeding. Unpaired Student $t$ tests were performed for comparing the plasma lipid levels of animals with different genotypes. Correlation studies were done using Kendall's rank-correlation tau test.

\section{Results}

Responses of plasma lipid levels to the atherogenic diet. All animals survived the 12 wk of diet feeding, except for homozygotes deliberately sacrificed after 6 wk of feeding. At the end of this period, the average gain in body weight was around 5 $\mathrm{g}$ for each animal and did not differ between sex or genotypes of the mice. The plasma cholesterol levels in all animals reached a plateau $\sim 2 \mathrm{wk}$ after initiation of the diet feeding and remained elevated throughout the $12 \mathrm{wk}$ feeding period (Fig. 1). Although the plasma cholesterol levels in heterozygotes and normal mice are the same $(P>0.05)$ when they are on regular chow, the cholesterol levels in the heterozygous mice on the atherogenic diet were higher than those of normal mice throughout the whole period of feeding. As shown in Table 1, the atherogenic diet changed the plasma total cholesterol levels of normal mice 2.5 -fold, from $94 \mathrm{mg} / \mathrm{dl}$ to $238 \mathrm{mg} / \mathrm{dl}(P$ $\ll 0.001)$. In heterozygous mice, the change was greater, 3.5 fold, from $97 \mathrm{mg} / \mathrm{dl}$ to $326 \mathrm{mg} / \mathrm{dl}(P \ll 0.001)$. The difference of cholesterol levels $(238 \mathrm{mg} / \mathrm{dl}$ versus $326 \mathrm{mg} / \mathrm{dl}$ ) between normal and heterozygous mice after feeding the atherogenic diet was also significant $(P<0.05)$. The cholesterol levels of homozygous mutants showed a 4.6-fold change after feeding the atherogenic diet, from $588 \mathrm{mg} / \mathrm{dl}$ to $2712 \mathrm{mg} / \mathrm{dl}(P$ $\ll 0.001$ ). Besides the changes in cholesterol levels, there is also a $40 \%$ reduction of triglyceride level in the homozygotes after feeding the atherogenic diet. Because of the overwhelmingly high concentration of apo B-containing lipoproteins in the 
Table I. Effect of the Atherogenic Diet on Plasma Lipid Levels in apo E Mutant Mice

\begin{tabular}{llrrcc}
\hline \multicolumn{1}{c}{ Animals } & \multicolumn{1}{c}{ Diet } & Time & Total-CH & Triglyceride & HDL-CH \\
\hline \multirow{4}{*}{ Normals } & & $w k$ & $m g / d l \pm S D$ & $m g / d l \pm S D$ & $m g / d l \pm S D$ \\
& LF & 0 & $90 \pm 13$ & $68 \pm 20$ & $62 \pm 18$ \\
& $n=10$ & 12 & $90 \pm 26$ & $52 \pm 7$ & $66 \pm 11$ \\
& HF & 0 & $94 \pm 13$ & $48 \pm 31$ & $77 \pm 11$ \\
& $n=11$ & 12 & $238 \pm 75$ & $53 \pm 41$ & $53 \pm 17$ \\
Heterozygotes & LF & 0 & $74 \pm 4$ & $95 \pm 20$ & $61 \pm 13$ \\
& $n=10$ & 12 & $77 \pm 12$ & $81 \pm 16$ & $53 \pm 2$ \\
& HF & 0 & $97 \pm 17$ & $69 \pm 28$ & $74 \pm 17$ \\
& $n=12$ & 12 & $326 \pm 153$ & $53 \pm 19$ & $56 \pm 22$ \\
Homozygotes & LF & 0 & $541 \pm 220$ & $133 \pm 38$ & $43 \pm 17$ \\
& $n=10$ & 12 & $672 \pm 284$ & $104 \pm 24$ & $37 \pm 14$ \\
& HF & 0 & $588 \pm 207$ & $105 \pm 60$ & $45 \pm 15$ \\
& $n=7$ & 12 & $2712 \pm 768$ & $64 \pm 29$ & ND \\
& & & & & \\
\hline
\end{tabular}

Results are means \pm SD of fasting plasma. $P$ values associated with the plasma lipid levels are discussed in the text. $L F$, regular chow; $H F$, atherogenic diet; $C H$, cholesterol; $T G$, triglyceride; $N D$, not determined.

plasma of these homozygous mutants after high-fat diet, the level of HDL cholesterol could not be determined reliably using the $\mathrm{MgCl}_{2}$-dextran sulfate precipitation assays.

Agarose gel electrophoresis (Fig. 2) shows an increase in lipid-staining lipoproteins in all the animals in response to the atherogenic diet, largely as the result of an increased level of $\beta$ - and pre- $\beta$-migrating particles. The intensity of lipid staining of $\alpha$-migrating particles indicates that the amounts of these particles in the normal and heterozygous mice were not changed significantly after feeding the atherogenic diet, but their nature appears to be altered; thus the $\alpha$-migrating particles in animals fed the atherogenic diet migrated faster and more diffusely than those in mice fed regular chow. In contrast, the amount of $\alpha$ migrating particles in homozygotes was reduced so profoundly that they could not be detected in agarose gels (Fig. 2).

These data show that an increased amount of $\beta$ - to pre $-\beta$ -

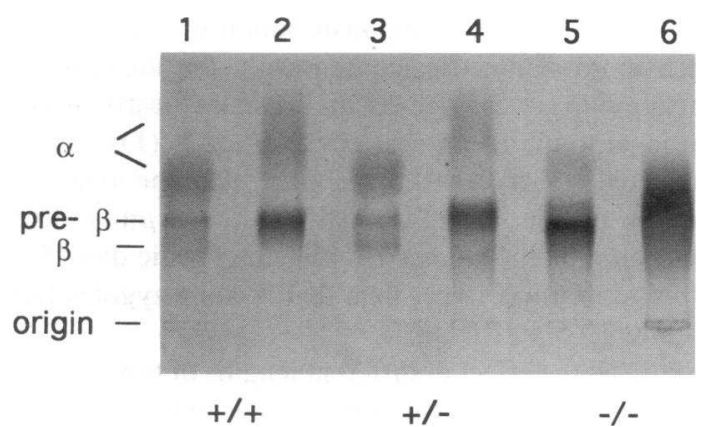

Figure 2. Agarose gel electrophoresis of lipoproteins in fasting plasma from mice on the atherogenic diet and the regular chow. Lanes 1, 3, and 5 are pooled plasma from mice fed regular chow. Lanes 2,4 , and 6 are pooled plasma from mice fed an atherogenic diet. The positions where the $\alpha$ (high density lipoprotein) particles, Pre- $\beta$ (very low density lipoprotein) particles, and $\beta$ (low density lipoprotein) particles normally migrate are labeled. $+/+$, normal mice; $+/-$, heterozygous apo $\mathrm{E}$ mutants; $-1-$, homozygous apo $\mathrm{E}$ mutants. The gel was stained for lipids with Oil Fat 7B.
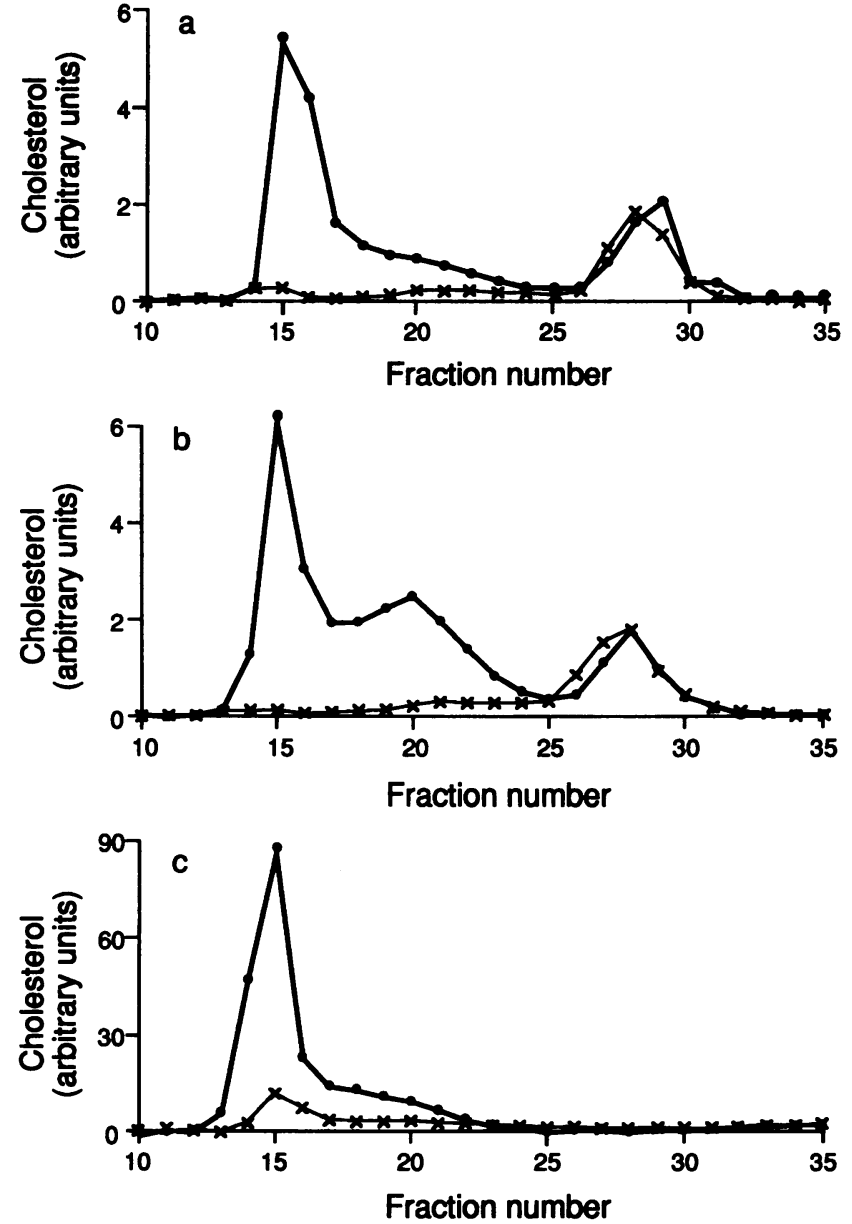

Figure 3. Lipoprotein profiles of mice revealed by FPLC. Crosses and dots indicate profiles of animals fed regular chow and atherogenic diet, respectively. ( $a$ ) Normal mice, $(b)$ heterozygotes, and $(c)$ homozygotes. Note that the vertical scale used in the profile for homozygotes $(c)$ is different from those used for normal mice $(a)$ and for heterozygotes (b). A total of 40 eluted fractions were assayed for cholesterol levels across each of these elusions.

migrating particles is the general response of mice of all three genotypes to the atherogenic diet. The amounts of $\beta$ - to pre $-\beta$ migrating particles that accumulate in normal and heterozygous mutant mice do not differ significantly. However, in the homozygous mutants, the accumulation of $\beta$ - to pre- $\beta$-migrating particles is considerably greater, and is accompanied by a decrease of both triglyceride and HDL cholesterol levels.

Changes in lipoproteins revealed by FPLC analysis. The different classes of plasma lipoproteins were separated by FPLC (Fig. 3). When mice were fed regular chow, HDL (peaking at around fraction number 28) was the major lipid-containing particle in both normal and heterozygous mice. After $2 \mathrm{wk}$ of feeding the atherogenic diet, the lipid profiles of both normal mice and heterozygotes showed no change in HDL particles, but there was a significant increase in the particles in fractions 13 to 23 , which normally include very low density lipoproteins (VLDL) and low density lipoproteins (LDL). There are severalfold more LDL particles in the heterozygotes fed the atherogenic diet than in normal mice on the same diet, as judged by the areas under the curve in the region (fractions 17 to 23) corresponding to the LDL fraction; this difference is sufficient 


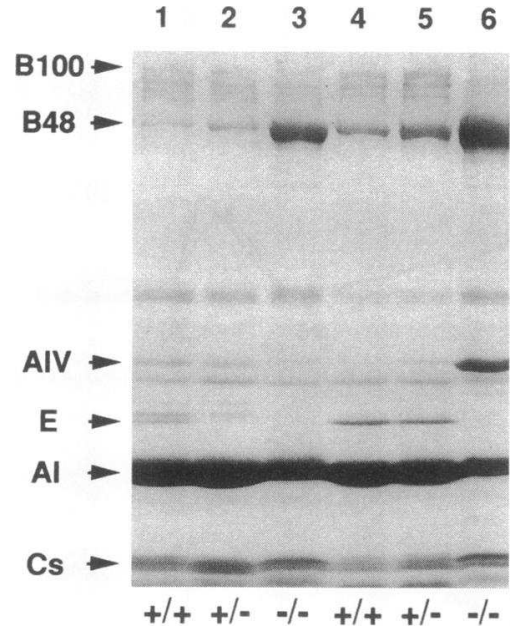

Figure 4. Apolipoprotein compositions of plasma total lipoprotein from mice fed regular chow and atherogenic diet. Total lipoproteins (from 40 $\mu l$ pooled plasma) isolated by density ultracentrifugation $(d>1.21 \mathrm{~g})$ $\mathrm{ml}$ ) were subjected to 4 $20 \%$ gradient SDS-polyacrylamide gel electrophoresis. Lanes 1-3 are from mice on regular chow, lanes 4-6 are from mice on atherogenic diet.

to account for the higher cholesterol levels in the heterozygous animals. In homozygotes, $12 \mathrm{wk}$ of the atherogenic diet resulted in extreme changes in the levels of particles in the VLDL-LDL size range, changing from 5 times normal on the regular chow to $\sim 12$ times normal on the atherogenic diet. The HDL cholesterol level of homozygous mutants on high-fat diet was reduced to one sixth of that of the similar mice on regular diet as measured by separating HDL particles from lipoproteins in the $1.019-1.346 \mathrm{~g} / \mathrm{ml}$ density range using Superose 6 column.

These various FPLC changes in lipid profiles of animals fed the diet are in agreement with the changes shown by agarose gel electrophoresis. The FPLC analysis offers the additional information that the greatest difference between the normal mice and heterozygotes in response to the atherogenic diet is that the LDL fraction of the heterozygotes increases several fold more than it does in normal animals.

Plasma apolipoprotein analyses. To further understand the changes of each apolipoprotein in response to the atherogenic diet, total plasma lipoproteins isolated by ultracentrifugation were subjected to SDS-PAGE (Fig. 4). Note that the levels of the various apolipoproteins are steady state levels; the relative contributions to these levels of synthesis, clearance etc. have not yet been determined. Densitometric scanning reveals a 10 times increase in the amount of apo B-48 (lanes 4-6), in animals of all three genotypes after feeding the atherogenic diet, without any significant changes in the amounts of apo B-100. There is also a very marked increase in the amount of apo AIV in the homozygous mutants (lane 6); a separate SDS-PAGE gel of VLDL and the intermediate density lipoproteins (IDL) shows that apo A-IV and apo B-48, as well as apo A-I are associated with the lipoproteins in these density ranges, which include the remnants of chylomicrons and VLDLs (data not shown). The amount of apo E associated with lipoproteins in heterozygotes fed the regular chow (lane 2) is about half of that seen in normal mice (lane 1). After feeding the atherogenic diet, the steady state levels of apo $\mathrm{E}$ in both heterozygous (lane 5 ) and normal mice (lane 4) increase substantially and become approximately equal as judged by protein staining in the gel. The same pattern of changes was also seen when the apo $E$ in whole plasma was visualized by Western blots (data not shown). The levels of apo A-I in the total plasma lipoproteins (lanes 3 and 6) appeared to be somewhat reduced in homozygotes on both diets. However, we suspect that this reduction is not real; it may be due to our loading slightly less sample in

the SDS-PAGE gel for the homozygotes, because of the effects on the Lowry assays of the large amount of lipid in these samples; or it may be caused by dissociation of apo A-I from the large lipoproteins (remnants of chylomicrons and VLDL) in these samples during the ultracentrifugation. In agreement with these possibilities is that the amount of apo A-I in whole plasma, as revealed by nonreducing SDS-PAGE (data not shown), did not differ among animals with different genotypes or on different diets. However, the distribution of apo A-I was different between homozygotes and normal as determined by densitometry scanning of the SDS-PAGE gel: only about $6 \%$ of apo A-I in homozygotes was associated with HDL while the rest of it was associated with larger lipoproteins with sizes of VLDLIDL, on the contrary, in normals $98 \%$ of apo A-I was with particles at HDL range.

These data therefore show that apo B-48 rich lipoprotein particles accumulate in mice of all three genotypes fed the atherogenic diet. The steady state apo E levels in heterozygotes, increase to about the same levels as those in normal mice after feeding the diet, even though the former is about half of those in normal mice when they are fed the regular chow. In homozygotes, unlike the normal and heterozygous mice, after feeding the atherogenic diet, besides apo B and apo C's, apo A-I and apo A-IV are also associated with particles having the density of VLDL and chylomicron remnants.

Morphometric evaluation of arterial lesions. We found no atherosclerotic lesions in the normal or heterozygous apo Edeficient mice fed regular chow. After the atherogenic diet, three out of nine normal mice developed fatty streak lesions, but they were quite small. In contrast, all nine heterozygotes developed substantial atherosclerotic lesions in the proximal aorta near the aortic valve attachment sites. The average (arithmetic mean) size of the atherosclerotic deposits in the nine heterozygotes $\left(1.3 \times 10^{4} \pm 1.6 \times 10^{4} \mu \mathrm{m}^{2}\right)$ was 30 times larger than the average size measured in the 9 normal mice $(420 \pm 570$ $\mu \mathrm{m}^{2}$ ). The average lesion size in the homozygous mutant mice fed the atherogenic diet for $12 \mathrm{wk}\left(5.3 \times 10^{5} \pm 2.4 \times 10^{5} \mu \mathrm{m}^{2}\right)$ was 15 times larger than in homozygotes of the same age fed the regular chow $\left(3.4 \times 10^{4} \pm 2.0 \times 10^{4} \mu \mathrm{m}^{2}\right)$.

When we plotted the lesion sizes of individual animals on a linear scale, we observed a highly asymmetric distribution of sizes; consequently their arithmetic mean greatly over represents the largest lesions. When the data are plotted on logarithmic scale as shown on Fig. 5, the distribution of lesion sizes appears much more symmetric; consequently the logarithmic mean of each group gives each animal a more nearly equal weight. The logarithmic mean size of the plaques (Fig. 5) in heterozygotes $\left(6,010 \mu \mathrm{m}^{2}\right)$ is 10 times larger than the logarithmic mean of the plaque size in normal mice $\left(630 \mu \mathrm{m}^{2}\right)$. The logarithmic mean in homozygotes fed the atherogenic diet $(5.2$ $\times 10^{5} \mu \mathrm{m}^{2}$ ) was 20 times larger than that in homozygotes fed the regular chow $\left(2.5 \times 10^{4} \mu \mathrm{m}^{2}\right)$.

We also studied the effect of different lengths of time of diet feeding on the development of the aortic lesions in homozygous mutants, as determined by percentage occlusion of the proximal aorta at the aortic sinus level. Lesions from homozygotes fed the atherogenic diet for 6 and 12 wk were compared. The occlusion from animals fed the atherogenic diet for 12 wk $(34.7 \pm 7.9 \%)$ was twice that of those fed for $6 \mathrm{wk}(15 \pm 3.9 \%)$. But, even the 6 wk of feeding the atherogenic diet generated lesions that were 10 times larger than those seen in age-matched homozygotes fed regular chow $(1.3 \pm 0.5 \%)$.

These morphometric observations clearly demonstrate that 


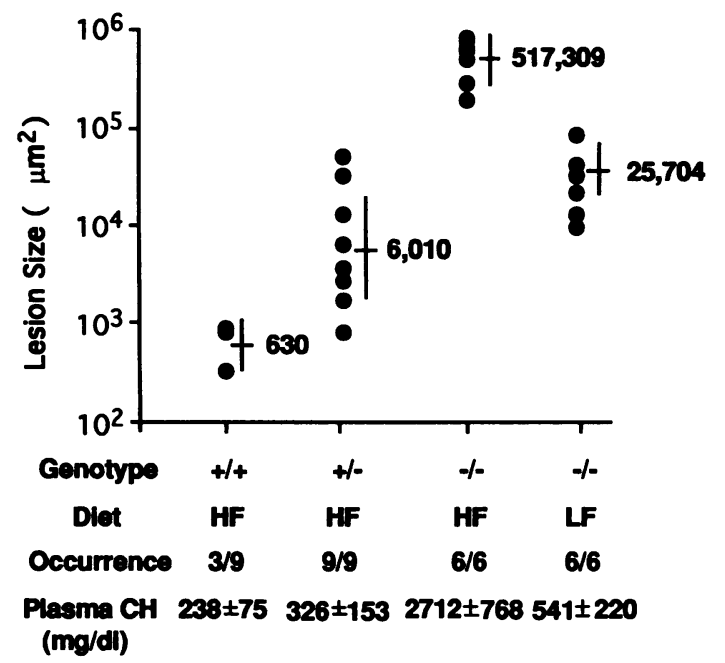

Figure 5. Morphometric evaluation of atherosclerotic lesion size in the aortic sinus and proximal aorta of mice fed atherogenic diet. Each point represents the mean lesion size of the four sections measured in each mouse (note that the vertical axis is logarithmic). The horizontal bars and the numbers beside them represent the logarithmic mean value in $\mu \mathrm{m}^{2}$ of lesion size from each group of mice. The vertical bars represent the range of logarithmic mean $\pm S D$ in logarithmic scale. Genotypes: +1 + , normal mice; $+/-$, heterozygotes; $-I-$, homozygotes. Diets: $H F$, atherogenic diet; $L F$, regular chow. Occurrence, number of mice with atherosclerotic lesions/total number of mice studied. Plasma $\mathrm{CH}$, plasma cholesterol levels in $\mathrm{mg} / \mathrm{dl}$ expressed as mean \pm SD. All the animals were 5-7 mo old. In the high fat groups, all the animals were fed the diet for $12 \mathrm{wk}$.

heterozygous apo E mutants are much more susceptible to dietinduced atherosclerosis than normal mice as judged by the occurrence and sizes of the lesions, and that the atherogenic diet dramatically accelerates the development of atherosclerotic lesions and their time course in homozygous apo E mutant mice as judged by the degree of arterial occlusion.

Lack of correlation between lesion size and plasma cholesterol levels. The large standard deviations in the data presented in Table $I$ and in Fig. 5 emphasize that there are considerable individual differences in plasma cholesterol levels and in the sizes of the atherosclerotic lesions developing in mice, particularly in heterozygotes, after $12 \mathrm{wk}$ of the atherogenic diet. The average lesion size ranged 65 -fold (from $780 \mu \mathrm{m}^{2}$ to 51,000 $\mu \mathrm{m}^{2}$ ) in the heterozygotes and fourfold (from 192,000 to $825,000 \mu \mathrm{m}^{2}$ ) in the homozygotes. There are obvious correlations between mean cholesterol levels, mean lesion sizes and apo $\mathrm{E}$ genotypes of mice fed either the regular chow or the atherogenic diet (Fig. 5). However, when individual animals within each group are examined, we could find no significant correlations between lesion sizes and plasma cholesterol levels, as judged by the Kendall's rank-correlation $\tau$ test (heterozygotes: $\tau=0.39, P=0.1$; homozygotes: $\tau=-0.5, P=0.1$ ).

Histologic evaluation of arterial lesions. Histologically, all the diet-induced lesions in normal and most of the lesions in the heterozygous mutant mice were early fatty streaks, ranging from superficial clusters of foam cells to layers of foam cells within the intima (Fig. $6 a$ ). However, fibrous cap formation was seen in one heterozygote, which also had the largest lesion size. In mice homozygous for apo E deficiency, the exacerbated progression of atherosclerotic lesions shows not only as a larger size with substantial arterial occlusion (Fig. $6 b$ ), but also as an increase in complexity (Fig. 6, $c$ and $d$ ) and a more extensive distribution along the arterial tree. For example, the lesions from 5-mo-old homozygotes fed regular chow consisted mainly of fatty streaks with few smooth muscle cells (data not shown), and the lesions were mainly confined to the proximal aorta and the aortic arch. However, after the atherogenic diet, the arterial lesions from an age-matched homozygote (Fig. $6 c$ ) consisted of multilayered foam cells admixed with smooth muscle cells, with calcification and inflammatory cells. Partial to complete occlusions of coronary arteries were commonly seen (Fig. 6 $d$ ), as well as partial to complete occlusions of pulmonary arteries, carotids, and iliac arteries.

These data document the development of fulminate atherosclerosis in the mice lacking apo $\mathrm{E}$ and clearly demonstrate that the mice are highly susceptible to the atherogenic diet. The dietinduced changes in area and complexity of the lesions in the heterozygous mutants shows that even partial apo E deficiency causes an increased susceptibility to the atherogenic diet.

Macroscopic and microscopic evaluations of other tissues in diet-fed animals. Macroscopic and microscopic examinations were made on tissues from nine normal mice, nine heterozygotes, and eight homozygotes fed the atherogenic diet for 12 wk and from four homozygous mutant mice fed the atherogenic diet for 6 wk.

Macroscopically, all mice were seen to have developed fatty livers and gall bladder stones. In the homozygotes, white, cheese-like lipid depositions were also visible to the naked eye on external surfaces of the kidneys, stomach, and the small and large intestines.

Microscopic evaluations of the normal mice and heterozygous mutants after $12 \mathrm{wk}$ on the atherogenic diet revealed no additional abnormalities, other than accumulation of lipid in hepatocytes and minor depositions of cutaneous lipid-filled macrophages (Feingold, K. R., S. H. Zhang, and N. Maeda, unpublished data). Histologic examination of the cheese-like depositions on the surfaces of various organs from the homozygotes showed that these lesions are composed of lipid-filled macrophages with admixed cholesterol clefts. Fig. $6 e$ shows an example of the histology of the lipid depositions on the external surface of the large intestine; the submucosa was not affected. But interestingly, lipid deposits were seen in the submucous space of the esophagus (Fig. $6 f$ ). The nature of the fatty liver observed in homozygotes after feeding the atherogenic diet was revealed by transmission electron micrography. Numerous lipid droplets are present in hepatocytes, as well as in macrophages between the hepatocytes and within the sinusoids (Fig. $7 a$ ). In the lungs of the diet-fed homozygous mutants, lipid-filled macrophages were seen in the interstitial spaces and in the vascular spaces (Fig. $7 b$ ).

These data show that, after feeding the atherogenic diet, massive amounts of lipid-loaded macrophages, are deposited outside the cardiovascular system in the apo E-deficient mice, in addition to their intravascular deposition.

\section{Discussion}

The data we have presented here show that in response to an atherogenic diet (consisting of $15.8 \%$ fat, $1.25 \%$ cholesterol, and $0.5 \%$ cholate) plasma cholesterol levels increased $\sim 2.5$ fold in normal mice, 3.5-fold in heterozygous mutants, and 5fold in homozygous apo E-deficient mice. Very large amounts of apo B-48-containing, $\beta$-migrating particles were present in the plasma of animals of all three genotypes after feeding the 

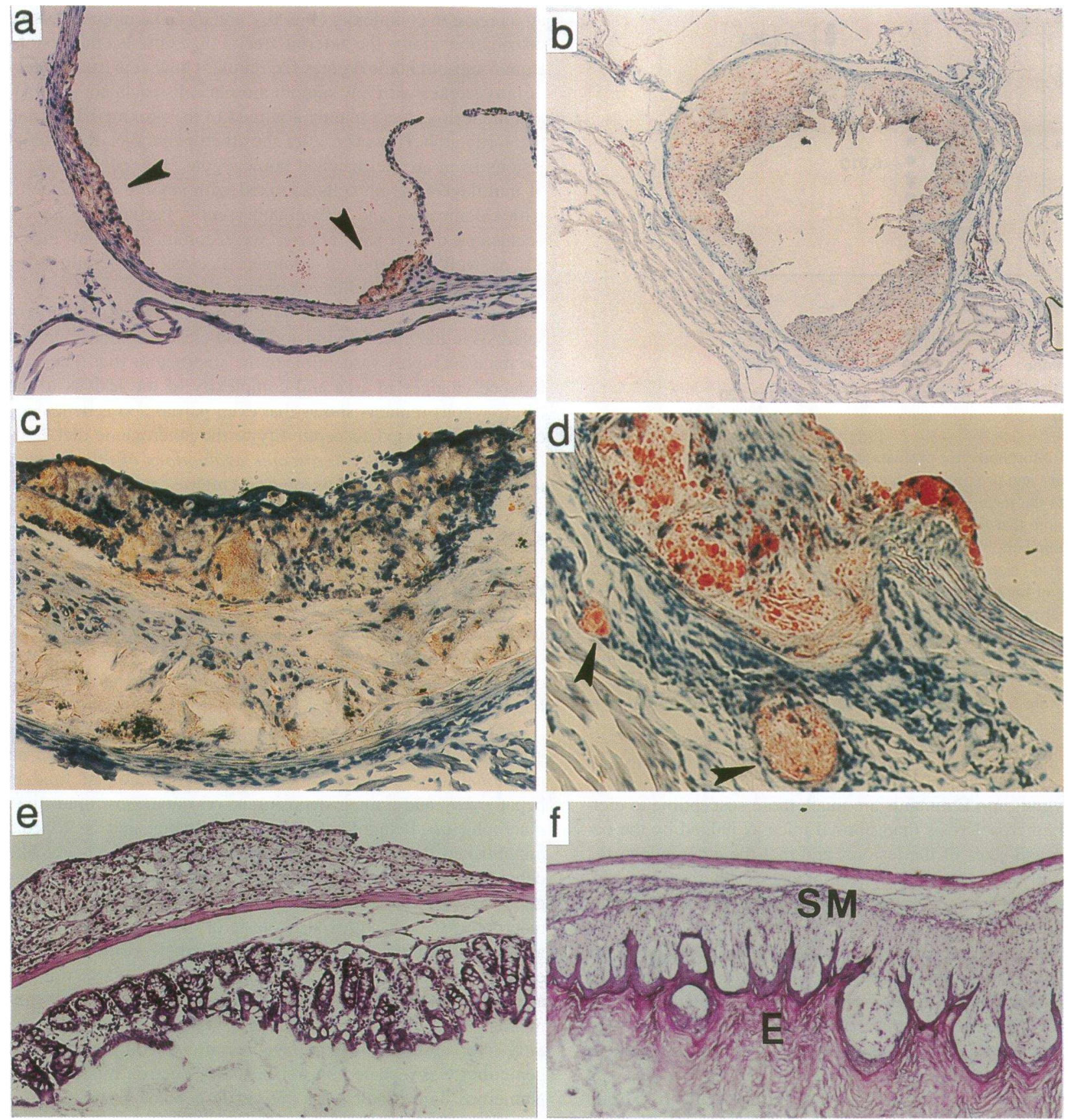

Figure 6. Pathological evaluations of arterial lesions and lipid depositions of organs after the atherogenic diet feeding. (a) Cross-section of the aortic sinus from a 20 -wk-old male heterozygous mouse, fed the diet for $12 \mathrm{wk}$. Arrows indicate early atherosclerotic lesions, which are mainly composed of foam cells. Cryosection, Sudan IV stain, Hematoxylin counter stain $(\times 255)$. (b) Cross section of the aortic sinus from a 30-wk-old male homozygote, fed the diet for $12 \mathrm{wk}$. The multilayered lesion caused a $61 \%$ occlusion of the vessel. Cryosection, Sudan IV stain, Hematoxylin counter stain $(\times 51)$. (c) Higher magnification of a lesion in the aortic sinus from a 20-wk-old male homozygote, fed the diet for 12 wk. The lesion includes foam cells, acellular lipid cores, admixed smooth muscle cells, calcification, inflammatory cells, and cholesterol clefts. Wall-thinning of the vessel is also seen. Cryosection, Sudan IV stain, Hematoxylin counter stain $(\times 437)$. (d) Higher magnification of a lesion in the aortic sinus from a 24-wk-old male homozygote, fed the diet for $6 \mathrm{wk}$. Coronary arteries included in the section were completely occluded by the plaque (arrows). Thinning and disruption of the elastic lamina in the vessel wall and reactive inflammatory cell accumulation around the lesion are also seen. Cryosection, Sudan IV stain, Hematoxylin counter stain $(\times 437)$. (e) Section from a 20-wk-old female homozygote fed the diet for 12 wk to illustrate lipid deposits on the outer surface of the large intestine. Cryosection, $\mathrm{H} \times \mathrm{E}$ stain $(\times 437)$. $(f)$ Section of the esophagus from a 20-wkold female homozygous mouse fed the diet for $12 \mathrm{wk}$. $E$, squamous epithelium; $S M$, submucosa. Cryosection, $\mathrm{H} \times \mathrm{E}$ stain $(\times 437)$.

diet. FPLC chromatography indicated that the majority of these particles are in the VLDL range. A notable difference between normal and heterozygous mutant mice after feeding the atherogenic diet was a several fold accumulation of particles in the
LDL range in the heterozygotes. Much larger atherosclerotic lesions were induced by the atherogenic diet in the heterozygotes than in normal mice. In the homozygous mutants, the development of atherosclerotic lesions was markedly acceler- 

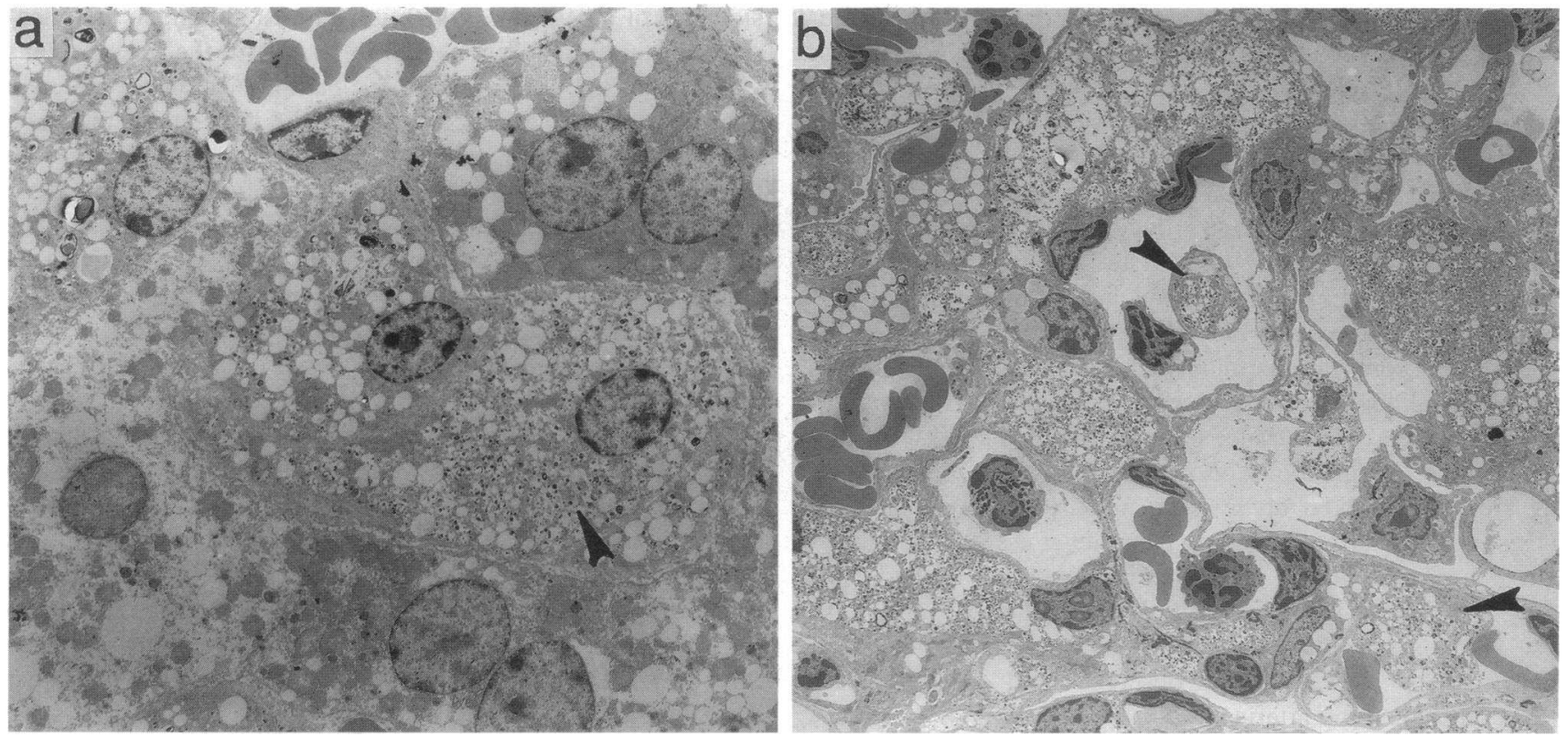

Figure 7. Transmission electron microscopy of the liver and the lung from a 20-wk-old female homozygous mouse fed the atherogenic diet for 12 wk. (a) Lipid-filled macrophages are present in the liver sinusoids (arrow); small lipid inclusions are also seen in the hepatocyte cytoplasm $(\times 1,950)$. (b) Lipid-filled macrophages are present within the interstitial spaces of the lung; occasional lipid-filled cells are also present within vascular spaces (arrows, $\times 940)$.

ated by the diet, as shown by its time course, and by the increases in size and in complexity. Thus, the mean lesion size in 5-7-mo-old homozygous mutant mice fed the atherogenic diet for $12 \mathrm{wk}$ is $\sim 30$-fold more on an arithmetic scale than in age-matched mutants maintained on regular chow, and 10fold more on a logarithmic scale. Cholesterol clefts and calcification, which become common features in mutants older than 10 mo when fed regular chow (10), are present in 5-7-mo-old mice that have been fed the atherogenic diet.

Results similar to ours with the mutant homozygotes have been reported by Plump and co-workers $(9,26)$, who fed homozygotes a Western-type diet ( $21 \%$ fat, $0.15 \%$ cholesterol ). They showed that, after $2.5 \mathrm{wk}$ of diet feeding, plasma cholesterol in the homozygotes increased to about four times the value seen in animals fed regular chow. Aortic lesion size after $4.5 \mathrm{wk}$ of diet feeding increased to about three times that of mice fed regular chow. The effects of diet on the induction of atherosclerosis in normal and heterozygous apo $\mathrm{E}$ mutant mice was not reported in their study. However, both studies clearly demonstrate that plasma cholesterol levels and the sizes of atherosclerotic lesions in apo E-deficient mice are markedly increased by the atherogenic diets. The detailed nature of the atherosclerotic lesions in the two studies are, unfortunately, not comparable since the duration of diet feeding, the lipid contents of the diet, and the ages of the mice were different in the two studies.

The dramatic acceleration in the development of atherosclerosis in homozygous apo $\mathrm{E}$ mutants that we report here is not surprising, considering that they are hypercholesterolemic and develop atherosclerotic lesions $(8-10)$ even when they are fed regular chow (4.5\% fat and $0.022 \%$ cholesterol). Since apo E functions as a ligand for receptors that mediate the clearance of the remnant particles of chylomicrons and VLDL, we expected to be able to correlate the atherogenic effects of the atherogenic diet with the lack of this clearance function. This correlation can be seen by examining our data from agarose gel electrophoresis, from FPLC analysis of plasma lipoprotein profiles, and from SDS-PAGE of plasma total lipoproteins. Combining the information from all three methods, we conclude that a huge amount of lipid carrying particles accumulates in these animals; these particles are cholesterol rich, which indicates that they are the remnants of chylomicrons and VLDL. The greatly increased amount of the particles is the most likely cause of the acceleration of atherosclerosis that we observe in these animals lacking apo E. However, the very large VLDL and chylomicron remnants which exist in the high fat fed apo E mutant mice in substantial amount, are probably less atherogenic than smaller lipoproteins because they do not enter the artery wall as effectively. This may be one of the contributing factors for the observed poor correlation in individual animals between the degree of lipid elevation and the severity of lesions in our study. Similar hypothesis was used to explain why diabetic rabbits, even at an equal total cholesterol level, get less atherosclerosis than non-diabetic rabbits on the same atherogenic diet (27); it is because that the non-diabetic rabbits have a lot more of their cholesterol in large VLDL or chylomicron remnants. Our biochemical studies also show that the $\alpha$-migrating particles, which are mostly HDL, are diminished to an almost undetectable level in response to the atherogenic diet, although the total plasma apo A-I content does not change. This suggests that apo A-I, which is a normal component of chylomicrons and VLDL particles, cannot leave the remnant particles in the absence of apo $\mathrm{E}$ and thus limits the HDL production. Therefore, in the apo $\mathrm{E}$ deficient mice, since the main apo A-I-containing lipoproteins are with the sizes of VLDL-IDL, but not HDL, the apo A-I level is not parallel to the level of HDL any more. Similarly, we find that Apo AIV, the majority of which is usually free in the plasma, also accumulates on the remnants of VLDL and chylomicrons. Both these events can contribute to the almost undetectable levels of HDL cholesterol that we observed. These very low levels of 
plasma HDL cholesterol would also be expected to contribute to the acceleration of atherosclerosis in the diet-fed homozygous mutants.

The unusual tissue distribution of lipids in the diet-fed homozygous mutants was very striking. We observed lipid-filled macrophages in the subendothelial space of the aorta, and also in the interstitial spaces of liver, lung, and skin tissues. Fatty deposits were even seen on the outer surfaces of various internal organs. The general histologic appearance of these tissues suggests that the atherogenic diet combined with the apo E deficiency of the homozygous mice causes macrophages to ingest lipids and/or lipoproteins vigorously. One hypothesis to explain this is that a delayed clearance of chylomicron and VLDL remnants causes modifications of the particles to the extent that they are readily taken up by scavenger receptors on macrophages (28). Clearly, the atherogenic diet stress greatly aggravates this process in the apo E-deficient mice. The markedly reduced HDL levels and possibly the lack of apo E per se in these animals may hamper the reverse cholesterol transport, which may be relevant to the observed lipid depositions in tissues other than the arteries.

Heterozygous mice, unlike the homozygous mutants, have plasma cholesterol levels and lipoprotein profiles indistinguishable from normal mice when both are fed regular chow. On the atherogenic diet, however, we found that the level of plasma cholesterol in heterozygotes was 1.6 fold that of normal mice. This increase was due to a several fold higher level of particles eluting in the LDL fractions in heterozygotes compared to the level seen in normal mice on the same diet. We observed a dramatic increase in plasma levels of apo B-48 and apo E in normal mice and on heterozygous mutants fed an atherogenic diet. Ishida et al. (29) have also observed a similar increase in both proteins in the plasma of normal animals. The amount of apo $\mathrm{E}$ in the plasma of normal mice and of heterozygotes increased substantially. Surprisingly, the final levels are the same in both animals fed the atherogenic diet, even though the heterozygotes have only one functional copy of the apo $\mathrm{E}$ gene. This phenomenon is understandable if the clearance from plasma of apo B-containing particles becomes limited and controlled by the plasma level of apo $\mathrm{E}$ in animals fed the atherogenic diet. Since the flux of the apo B-containing particles must be the same in the normal and heterozygous mice once the steady state is reached, the apo E plasma levels at this steady state should also be the same in both normal and heterozygous mice. But the synthesis of apo $\mathrm{E}$ in heterozygotes is most likely half that of normal mice. We therefore expect the level of apo Bcontaining particles in the heterozygotes to stabilize at a level double that of the normal mice, therefore, the transit time of these particles must be twice as long in the heterozygotes. Thus, the prolonged circulation of apo E/apo B-containing lipoproteins in the heterozygotes is the most likely explanation for the increased susceptibility of these heterozygous animals to atherosclerosis. These data also suggest that the plasma level of apo $\mathrm{E}$ by itself is likely to be a poor indicator of risk of atherosclerosis under the atherogenic diet stress. The ratio of apo E/apo B appears likely to be a much better index.

The most important new finding in our current experiments is the consistent induction of atherosclerotic lesions in heterozygotes carrying one normal and one disrupted apo E gene. Only 3 out of 9 normal mice fed the atherogenic diet for 12 wk developed lesions, with a logarithmic mean size of $630 \mu \mathrm{m}^{2}$. In contrast, all of the 9 heterozygotes fed the atherogenic diet developed atherosclerotic lesions with a logarithmic mean le- sion size of $6,010 \mu \mathrm{m}^{2}$, which is 10 times more than that measured in the normal animals. The lesions in the heterozygous animals fed the atherogenic diet obtain a size and completely equivalent to the lesions of homozygous mutants of the same age fed regular chow. The more frequent occurrence and the larger size of the atherosclerotic lesions in the heterozygotes compared to normal mice show that mice carrying only one normal apo $\mathrm{E}$ gene are much more susceptible to diet-induced atherosclerosis than are their normal littermates, although neither develop lesions when fed regular chow.

Our results emphasize the importance of using heterozygous mutants when studying the effects of environmental factors on atherogenesis, and when searching for their significance for human populations. Homozygous mice completely lacking apo $E$ have many valuable uses, including the study of the progression of atherosclerotic lesions and the effects of drugs on this progress. However, the number of humans carrying the equivalent genetic defect is so small $(30-32)$ that the responses to environmental factors of the apo $\mathrm{E}$ null mutants may not have direct relevance to the general human population. On the other hand, mice heterozygous for the disrupted apo E gene have essentially normal lipid biochemical parameters and are free from atherosclerotic lesions when they are on regular chow. Yet, upon dietary stress, delayed clearance of apo E containing particles was observed in the heterozygous mutants, accompanied by other lipid abnormalities and an increased susceptibility to atherosclerosis. The heterozygous mice should, therefore, be more valuable than the homozygotes for studying the relationships between dietary manipulation and coronary heart disease prevention. A quantitative genetic predisposition to diet-induced susceptibility similar to that revealed by our present work has been seen in heterozygous Watanabe heritable hyperlipidemic rabbits (33). It is reasonable to expect, therefore, that genetically determined quantitative limitations of apo $E$ will have similar effects in humans when they are stressed by an atherogenic diet, even though the same individuals may be asymptomatic on a high-fat, high-cholesterol diet. Relationships between apo $\mathrm{E}$ isoforms and plasma cholesterol levels or atherosclerosis has been emphasized in recent years. For example, in studies of the prevalence of different apo $\mathrm{E}$ isoforms in patients with myocardial infarction, the apo $\mathrm{E} 4$ allele was shown to be most prevalent, and the onset of coronary artery disease is earlier in individuals with the apo E4 allele than with other isoforms (34). It is of interest to note that Boerwinkle and Utermann have reported (35) that the apo E polymorphism in humans is one of the contributing factors in determining plasma levels of apo $B$ and apo E; for example, the apo B and apo E levels in apo E4/E4 homozygotes are the highest and the lowest respectively of those found in any of the isoforms.

Our observation that the logarithmic values of the lesion sizes of animals with the same genotype are more symmetrically distributed than the arithmetic values is of interest. We have shown previously (10) that the increase of lesion size with age is logarithmic in apo E-deficient mice fed regular chow. This suggests that the large variation in lesion size among animals, especially among the heterozygotes, is the result of differences in the times of initiation of the lesion, followed by a subsequent logarithmic growth in their size. Factors that determine this difference, either genetic or environmental, warrant further investigation.

In conclusion, we have investigated the interaction between a known genetic defect and a defined environmental stress in both homozygous and heterozygous apo E-deficient mice. Het- 
erozygous mice, with one normal and one genetically inactivated apo $\mathrm{E}$ allele, can compensate for the loss of one allele without developing atherosclerosis when fed a regular chow. But the heterozygotes are much more sensitive to high-fat dietinduced atherosclerosis than are normal mice. Homozygous mice, completely lacking apo E, are extremely sensitive to the atherogenic diet as shown by the extreme elevations of their plasma cholesterol levels and by the accelerated time course and the severity of their atherosclerosis. Thus both heterozygous and homozygous apo $\mathrm{E}$ mutants are in different ways very valuable models for studying atherosclerosis.

\section{Acknowledgments}

The authors sincerely thank Lara H. Kester, John B. Reynolds, Jr., Bruce Eskesen, and Xiaowen Ma for their excellent technical help. Thanks also go to Drs. Oliver Smithies, Jesus Osada, and Sarah Bronson for their valuable suggestions to the manuscript.

This work was supported by National Institutes of Health grant HL42630 to N. Maeda and by a Department of Pathology grant to R. L. Reddick.

\section{References}

1. Steinberg, D., and J. L. Witztum. 1990. Lipoprotein and atherogenesis: current concepts. JAMA (J. Am. Med. Assoc.) 264:3047-3052.

2. Hegele, R. A. 1992. Gene-environment interactions in atherosclerosis. Mol. Cell Biochem. 113:177-186.

3. Maeda, N. 1993. Gene targeting in mice as a strategy for understanding lipid metabolism and atherogenesis. Curr. Opin. Lipidol. 4:90-94.

4. Stoltzfus, L., and E. M. Rubin. 1993. Atherogenesis: insights from the study of transgenic and gene-targeted mice. Trends Cardiovasc. Med. 3:130-134.

5. Lusis, A. J. 1993. The mouse model for atherosclerosis. Trends Cardiovasc. Med. 3:135-143.

6. Breslow, J. L. 1993. Transgenic mouse models of lipoprotein metabolism and atherosclerosis. Proc. Natl. Acad. Sci. USA. 90:8314-8318.

7. Ishibashi, S., M. S. Brown, J. L. Goldstein, R. D. Gerard, R. E. Hammer, and J. Herz. 1993. Hypercholesterolemia in low density lipoprotein receptor knockout mice and its reversal by adenovirus-mediated gene delivery. J. Clin. Invest. 92:882-893.

8. Zhang, S. H., R. L. Reddick, J. A. Piedrahita, and N. Maeda. 1992. Spontaneous hypercholesterolemia and arterial lesions in mice lacking apolipoprotein E. Science (Wash. DC). 258:468-471.

9. Plump, A. S., J. D. Smith, T. Hayek, K. Aalto-Setala, A. Walsh, J. G. Verstuyft, E. M. Rubin, and J. L. Breslow. 1992. Severe hypercholesterolemia and atherosclerosis in apolipoprotein E-deficient mice created by homologous recombination in ES cells. Cell. 71:343-353.

10. Reddick, R. L., S. H. Zhang, and N. Maeda. 1994. Atherosclerosis in mice lacking apolipoprotein $\mathrm{E}$ : evaluation of lesional development and progression. Arterioscler. Thromb. 14:141-147.

11. Flynn, M. A., G. B. Nolph, G. Y. Sun, M. Navidi, and G. Krause. 1991. Effects of cholesterol and fat modification of self-selected diets on serum lipids and their specific fatty acids in normocholesterolemic and hypercholesterolemic Humans. J. Am. Coll. Nutrition. 10(2):93-106.

12. Thomas, W. A., and W. S. Hartroft. 1959. Myocardial infarction in rats fed diets containing high fat, cholesterol, thiouracil, and sodium cholate. Circulation XIX:65-72.

13. Zilversmit, D. B. 1979. Atherogenesis: a postprandial phenomenon. Circulation. 60:473-485.

14. Fainaru, M., R. W. Mahley, R. L. Hamilton, and T. L. Innerarity. 1982. Structural and metabolic heterogeneity of $\beta$-very low density lipoproteins from cholesterol-fed dogs and from humans with type III hyperlipoproteinemia. $J$. Lipid Res. 23:702-714.

15. Daugherty, A., K. Oida, B. E. Sobel, and G. Schonfeld. 1988. Dependence of metabolic and structural heterogeneity of cholesterol ester-rich very low density lipoproteins on the duration of cholesterol feeding in rabbits. J. Clin. Invest. 82:562-570.

16. Crook, D., K. H. Weisgraber, L. K. Boyles, and R. W. Mahely. 1990. Isolation and characterization of plasma lipoproteins of common marmoset monkey: comparison of effects of control and atherogenic diets. Arteriosclerosis. 10:633-647.

17. Miettinen, T. A., H. Gylling, and H. Vanhanen. 1988. Serum cholesterol response to dietary cholesterol and apoprotein E phenotype. Lancet. II:1261.

18. Tikkanen, M. J., J. K. Huttunen, C. Ehnholm, and P. Pietinen. 1990 Apolipoprotein E4 homozygosity predisposes to serum cholesterol elevation during high fat diet. Arteriosclerosis. 10:285-288.

19. Manttari, M., P. Koskinen, C. Ehnholm, J. K. Huttunen, and V. Manninen. 1991. Apolipoprotein E polymorphism influences the serum cholesterol response to dietary intervention. Metabolism. 40:217-221.

20. Hixson, J. E., and the Pathobiological Determinants of Atherosclerosis in Youth (PDAY) Research Group. 1991. Apolipoprotein E polymorphisms affect atherosclerosis in young males. Arterioscler. Thromb. 11:1237-1244.

21. Glatz, J. F. P. N. M. Demacker, P. R. Turner, and M. B. Katan. 1991. Response of serum cholesterol to dietary cholesterol in relation to apolipoprotein E phenotype. Nutr. Metab. Cardiovas. Dis. 1:13-17.

22. Savolainen, M. J., M. Rantala, K. Kervinen, I. Jarvi, K. Suvanto, T. Rantala, and Y. A. Kesaniemi. 1991. Magnitude of dietary effect on plasma cholesterol concentration: role of sex and apolipoprotein E phenotype. Atherosclerosis. 86:145-152.

23. Warnick, G. R., J. Benderson, and J. J. Albers. 1982. Dextran sulfate$\mathrm{Mg}^{2+}$ precipitation procedure for quantitation of high-density-lipoprotein cholesterol. Clin. Chem. 28(6):1379-1382.

24. France, D. S., R. B. Quimby, J. Babiak, D. C. Lapen, R. J. Paterniti, and D. B. Weinstein. 1990. Clinical chemistry applications of microplated robotic systems in a lipoprotein laboratory. Lab. Rob. \& Auto. 2:155-173.

25. Havel, R. J., H. A. Eder, and J. H. Bragdon. 1955. The distribution and chemical composition of ultracentrifugally separated lipoproteins in human serum. J. Clin. Invest. 55:1345-1353.

26. Nakashima, Y., A. S. Plump, E. W. Raines, J. L. Breslow, and R. Ross. 1994. Apo E-deficient mice develop lesions of all phases of atherosclerosis throughout the arterial tree. Arterioscler. Thromb. 14:133-140.

27. Brecher, P., V. A. Chobanian, D. M. Small, W. V. Sickle, A. Tercyak, A. Lazzari, and J. Baler. 1983. Relationship of an abnormal plasma lipoprotein to protection from atherosclerosis in the cholesterol-fed diabetic rabbit. $J$. Clin. Invest. 72:1553-1562.

28. Keidar, S., M. Kaplan, M. Rosenblat, G. J. Brook, and M. Avira. 1992 Apolipoprotein $\mathrm{E}$ and lipoprotein lipase reduce macrophage degradation of oxidized very-low-density lipoprotein (VLDL), but increase cellular degradation of native VLDL. Metabolism. 41:1185-1192.

29. Ishida, B. Y., P. J. Blanche, A. V. Nichols, M. Yashar, and B. Paigen 1991. Effects of atherogenic diet consumption on lipoproteins in mouse strains C57BL/6 and C3H. J. Lipid Res. 32:559-568.

30. Gabelli, C., R. E. Gregg, L. A. Zech, E. Manzato, and H. B. Brewer, Jr 1986. Abnormal low density lipoprotein metabolism in apolipoprotein $\mathrm{E}$ deficiency. J. Lipid Res. 27:326-333.

31. Mabuchi, H., H. Itoh, M. Takeda, K. Kajinami, T. Wakasugi, J. Koizumi, R. Takeda, and C. Asagami. 1989. A young type III hyperlipoproteinemic patient associated with apolipoprotein E deficiency. Metabolism. 38(2):115-119.

32. Kurosaka, D., T. Teramoto, T. Matsushima, T. Yokoyama, A. Yamada, T. Aikawa, Y. Miyamoto, and K. Kurokawa. 1991. Apolipoprotein E deficiency with a depressed mRNA of normal size. Atherosclerosis. 88:15-20.

33. Atkinson, J. B., R. L. Hoover, K. K. Berry, and L. L. Swift. 1989. Cholesterol-fed heterozygous Watanabe heritable hyperlipidemic rabbits: a new model for atherosclerosis. Atherosclerosis. 78:123-136.

34. Cumming, A. M., and F. W. Robertson. 1984. Polymorphism at the apoprotein-E locus in relation to risk of coronary disease. Clin. Genetics. 25:310313.

35. Boerwinkle, E., and G. Utermann. 1988. Simultaneous effects of the apolipoprotein $\mathrm{E}$ polymorphism on apolipoprotein $\mathrm{E}$, apolipoprotein $\mathrm{B}$ and cholesterol metabolism. Am. J. Hum. Genet. 42:104-112. 